\section{De las cosas}

\section{maravillosas.}

\section{Niños pensando sobre filosofar en la escuela}

\section{Das coisas maravilhosas. Crianças pensando sobre filosofar na escola}

\author{
Wonderful things. \\ Children thinking about doing \\ philosophy at the school
}

\section{Beatriz Fabiana Olarieta*}

* Profesora de Nivel Inicial por la ESFD de la Universidad Nacional de Cuyo (Argentina); profesora en Ciencias de la Educación por la misma universidad; especialista en Enseñanza de la Filosofía por la Universidad de Brasilia (Brasil), máster en Educación por la Universidad del Estado de Río de Janeiro; doctoranda en educación en la misma Universidad. Filiación institucional: Universidade do Estado do Rio de Janeiro (Brasil). Correo electrónico: olarietaf@hotmail.com

Este trabajo será publicado como capítulo de libro en: Kohan, Walter O. y Olarieta, B.F. (2012). A escola pública aposta no pensamento. Belo Horizonte: Autêntica (en proceso de edición).

\section{Resumen}

A partir de la conversación de un grupo de niños de Educación Básica, que desde hace dos años participa de los encuentros de filosofía realizados en el marco del proyecto de extensión del Núcleo de Estudos Filosóficos da Infância (NEFI) "Em Caxias, a filosofia em-caixa?", desarrollado en escuelas públicas del municipio de $\mathrm{Du}$ que de Caxias (Rio de Janeiro, Brasil), el artículo intenta repensar qué significa hacer filosofía en la escuela para aquellos que son sus protagonistas. Apropiándose de las cuestiones traídas por ellos, explora el sentido de ese tiempo y espacio diferenciados que cada semana convoca al grupo.

\section{Palabras clave}

Filosofía con niños, escuela, tiempo, Bergson.

\section{Resumo}

A partir da conversação com um grupo de crianças da Educação Básica, que há dos anos participa dos encontros de filosofia realizados no marco do projeto de extensão do Núcleo de Estudos Filosóficos da Infância (NEFI) "Em Caxias, a filosofia em-caixa?", desenvolvido em escolas públicas no município de Duque de Caxias (Rio de Janeiro, Brasil), o artigo pretende repensar que significa fazer filosofia na escola para aqueles que são seus protagonistas. Apropriando-se das questões trazidas por eles, explora o sentido desse tempo e espaço diferenciados que cada semana convoca ao grupo.

\section{Palavras chave}

Filosofia com crianças, escola, tempo, Bergson.

\section{Abstract}

From a conversation of a group of children from elementary school, that participates in the meetings of philosophy two years ago developed within the context of the extension project from the Center for Philosophical Studies of Childhood "Does Philosophy fit in Caxias?" (Rio de Janeiro, Brazil), this article intends to rethink what means to do philosophy in school to those who are its stars and, appropriating the questions raised by them, explores the meaning of a different time and space that summons the class each week.

\section{Key words}

Philosophy with children, school, time, Bergson.

Fecha de recepción: 16 de mayo de 2012 Fecha de aprobación: 2 de octubre de 2012

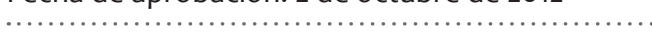


con la filosofía venía desenvolviéndose en la escuela. Además, el grado había sido repartido en dos grupos en los que se mezclaban con otros alumnos que, al igual que sus profesores, desconocían el proyecto. Esa condición, definitivamente, parecía insuperable. Pero los niños tampoco se dieron por vencidos. Continuaron pidiendo sus clases de filosofía. Flavia y yo estábamos dispuestas a continuar, pero fue imposible encontrar simultáneamente en los dos grados, una hora libre dentro del horario. La única opción en la que pudimos pensar fue en un horario después de clase. Los niños aceptaron entusiasmados. En los días de nuestros encuentros uno de los grados salía un poco antes y se quedaba esperando a los compañeros del otro curso para poder comenzar. Teníamos en cada encuentro un promedio de quince niños aproximándose vertiginosamente a la adolescencia, pero conservando la misma alegría infantil por el espacio conquistado.

¿Por qué esa insistencia? ¿Por qué esa obstinación por no renunciar al momento de la clase de filosofía? Creo hallar parte de la respuesta a mis preguntas en una conversación que se produjo en los primeros encuentros de ese ya avanzado 2009, cuando Flavia y yo volvíamos a acompañar al grupo después de las reiteradas solicitudes de los niños para retomar el trabajo. La respuesta no apareció en forma directa, apareció en la voz de algunos niños como intento de pensar otra cuestión planteada por ellos.

En esa ocasión, en medio de una discusión alguien preguntó: ¿Qué es la filosofía?. Sabemos que muchas de las cuestiones que surgen en los encuentros pasan sin que nadie se las apropie, a veces inclusive ante la insistencia de quien la formuló, es resistida. Pero esta pregunta por la filosofía rápidamente dejó de ser la pregunta de uno para transformarse en una cuestión del grupo. Los niños comenzaron a explorar lo que es la filosofía a partir de la propia vivencia de haber sido parte de ese ritual durante todo un año; en este ritual, los mismos niños que se encontraban durante cuatro horas de lunes a viernes en el aula se dirigían a la sala de filosofía por una hora semanal. Ritual que ahora habían conseguido retomar. A continuación, un pequeño extracto de lo que dijeron en aquella ocasión:

Matheus Augusto: Aquí en filosofía aprendemos y nos divertimos.

Le pregunto: ¿Y en el aula no aprenden?

Matheus Augusto [aclara]: En el aula aprendemos a pasar de grado estudiando. Aquí [se refiere al encuentro de filosofía] aprendemos a discutir, vamos sabiendo más cosas, vamos haciendo más preguntas.
Diogo [que hace solo tres encuentros que participa, se arriesga a definir]: Es una clase de conversación en la que aprendemos más. Si estamos con una duda le preguntamos a otro y van creciendo las dudas y las respuestas.

Paulo [no se conforma]: No es solo una clase de preguntas y respuestas. Nos divertimos, dibujamos, pintamos. Y mientras vamos haciendo eso las preguntas van aumentando.

Wesley [completa]: Las preguntas salen de nuestra mente. Vamos sacando las dudas de nuestra cabeza.

Ricardo: La filosofía es una conversación en la que llegamos al punto de las preguntas.

Flavia: ¿Qué significa "llegar al punto de una pregunta"?

Matheus Augusto [no le da tiempo a Diogo a responder y se arriesga]: Es aclarar dudas.

Wesley [reformula]: Cuando van viniendo más dudas.

Ricardo [quien acuñó la expresión, encuentra su momento para responder]: Que es más fácil juzgar las preguntas que tenemos en la cabeza. Acá hay gente ayudándome a llegar al punto de mi pregunta.

¿Y qué sería juzgar?, pregunto.

Ricardo: Juzgar es pensar más fácil con la pregunta. Si todo el mundo concuerda, ya llegamos al punto de esa pregunta.

Wesley [no se conforma]: Juzgar es aclarar las preguntas, discutir sobre ellas.

Yasmín [comparte su punto de vista]: Es aprender a aclarar las preguntas que tenemos y no sabemos.

Rodrigo [se arriesga a definir]: La clase de filosofía es una charla, nos quedamos conversando.

Matheus [se interesa por la idea]: Es como una conversación en el MSN (Messenger), donde las personas se quedan conversando.

Arthur [trae una comparación que le parece más adecuada]: La filosofía se parece al recreo, es nuestro recreo. Solo que aquí hacemos preguntas y en el recreo conversamos porque sí.

Matheus Augusto [se quedó pensando en la intervención de Matheus y dice]: Creo que la filosofía es diferente a la conversación en el MSN. Ahí uno habla 
de fútbol, de chicas... y aquí estamos aprendiendo a aclarar nuestras dudas. Los asuntos son diferentes. Alguien no va a preguntar en el MSN ¿por qué el agua es azul?

Aquí aprendemos cosas que ni imaginábamos. Por ejemplo, si estoy en mi casa mirando el cielo a la noche y veo una estrella fugaz, y me pregunto: “¿Por qué hay estrellas fugaces?”. ¿Dónde les parece a ustedes que voy a aclarar mi pregunta? ¿En el aula? ¡No! ¿En mi casa? ¡No! ¿En el MSN? ¡No! [Levanta los hombros y hace un gesto con las manos señalando el piso, como si fuera evidente que esa pregunta es para el espacio donde realizamos nuestros encuentros].

Wesley [no está tan de acuerdo]: Creo que sí podría sentarme a hacer filosofía con mi abuela, pero ¡seríamos solo dos y no se puede! Y en el MSN también se podría. ¡Podríamos hacer un MSN de filosofía y colocar nuestras preguntas para responderlas! [Algunos compañeros asienten y parecen entusiasmados con la idea].

Esther [que esperaba su turno para comentar la afirmación de Arthur]: Creo que la filosofía no tiene nada que ver con el recreo porque en el recreo no conversamos así.

Paulo [asiente y marca la diferencia]: Me parece que aquí los otros me ayudan a pensar. Yo pienso mejor con los otros.

Felipe [interviene]: Yo le quiero preguntar a Matheus Augusto si le parece que es posible hacer esas preguntas que él dice en la clase normal.

La respuesta de Matheus Augusto es rotunda: No. No hay cómo hacer preguntas allá. Allá hay que hablar. Las preguntas son sobre los deberes, para ver si entendí lo que hay que hacer. Allá pregunto y me informo. Aquí pregunto sobre lo que pensamos.

Sebastião [marca otra diferencia]: Lo que pasa es que aquí nos relajamos, pensamos, nos sacamos las dudas. Salimos de acá y no nos relajamos más.

Paulo [acota]: Acá es el momento en que solo dialogamos. No hay nadie interfiriendo.

Le solicito una aclaración a Paulo: ¿Por qué dices que no hay nadie estorbando si es la misma maestra del grado la que hacía filosofía con ustedes el año pasado, el grupo de compañeros era el mismo tanto aquí como en el aula. Acá hay otras personas que no están en el aula. En todo caso, podríamos pensar que acá hay algo que allá no hay y que eso que se agrega podría estorbar, no al contrario. ¿Cómo estás pensando esta cuestión? ¿Qué es lo que interfiere en el aula que acá no interfiere si somos prácticamente los mismos?

Ricardo [responde haciendo suya la pregunta]: Allá tenemos que investigar, copiar del pizarrón, hacer tareas, hacer cuentas... [Se queda pensando].

Paulo [asiente con la cabeza y no agrega nada más a las interferencias que Ricardo mencionó. Se limita a afirmar]: Aquí podemos hablar de verdad, sin que nada interfiera.

Wesley [insiste con la comparación con el aula]: Aprendemos más cosas que en el grado. Aquí tenemos tiempo libre para hablar y pensar. Es muy interesante tener preguntas y quedarnos con dudas.

Es llamativo ver cómo los chicos abundan en expresiones que tienen que ver con el tiempo y el espacio cuando tratan de pensar qué es lo que caracteriza un encuentro de filosofía. Perciben ese momento como un tiempo y un espacio diferentes: diferente del tiempo de clase, del tiempo del chat, del tiempo con la familia. Un tiempo que se aproxima en algo al del recreo o a un tiempo libre, sin interferencias, sin estorbos.

La filosofía para ellos toma cuerpo en una conversación colectiva que se inscribe en un tiempo percibido como fuera de lo ordinario, un tiempo que podríamos pensar como un tiempo extra-ordinario, fuera de lo habitual (tal vez por eso tenga algo parecido con el recreo) y que genera un tipo de comunicación distinta, o mejor, una forma que les permite inscribirse; explorar lo que les acontece, lo que los inquieta, lo que los afecta, lo que piensan, lo que son. Los niños hacen filosofía en la escuela, pero el momento de la filosofía parece alterar el tiempo habitual de la institución y la forma de habitarlo.

El tiempo de la escuela, desde una perspectiva tradicional, es un tiempo lineal y mensurable. Allí los niños son clasificados de acuerdo con su edad y distribuidos en espacios diferentes, en los cuales deberán desenvolver una serie de habilidades y apropiarse de conocimientos ordenados uniformemente a lo largo de un año, que les permitirán pasar de grado, avanzar al momento siguiente e inmediatamente superior. La vida escolar tradicional, por la necesidad de organizar el tiempo y el espacio de la institución y de quienes por ella transitan, muchas veces acaba empobreciendo la experiencia, reduciéndola al acontecimiento de lo ya previsto. Los niños y los propios maestros deberán adecuarse a los tiempos y a los contenidos ya planificados, pensados por otros, para que ellos se interesen en ese momento de sus vidas. 
Pueblan el vocabulario escolar palabras como planificación, objetivos, proyecto, logros, repitencia, adelantado, atrasado, deberes, pruebas, notas, éxito, fracaso. En la escuela el tiempo avanza o se atrasa, los conocimientos se acumulan o se pierden, se olvidan. En esa lógica, la maestra hace las preguntas para verificar los saberes adquiridos, y, a veces, el alumno, para verificar si comprendió bien las consignas del trabajo o los contenidos que le están siendo presentados y de los cuales deberá apropiarse y dar cuenta. Es difícil inscribir en esa forma de relacionarse con el tiempo la propia experiencia del mundo y trabajar sobre ella. Es eso lo que Matheus Augusto distingue. Al aula él no puede llevar "sus" preguntas porque, como él mismo dice, allá solo se pregunta para informarse o para entender lo que se debe hacer; pero las preguntas que él se hace, aquellas que lo inquietan, esas que le aparecen a la noche cuando contempla el cielo, no se satisfacen con la información. Es interesante notar cómo esa lógica escolar en muchas oportunidades está más allá de las personas particulares que la habitan. En el caso al cual estamos haciendo referencia, es la misma maestra del grupo quien coordinaba los encuentros de filosofía que se habían realizado durante todo un año. Es con ella y con sus compañeros con quien Matheus Augusto está conversando sobre las diferencias que él percibe entre las clases normales (como las denomina Felipe) y los encuentros de filosofía. El mismo grupo de niños y la misma maestra funcionan de un modo diferente cuando se apartan, por un momento, de una forma de lidiar con la realidad en términos de contenidos y objetivos, fracasos y logros, avances y retrocesos, y números que pretenden cuantificar la experiencia.

En los encuentros de filosofía los niños distinguen que la lógica es otra. Mientras en el salón de clases preguntan para informarse, aquí, en las palabras de ellos, pueden hacer "esas preguntas que tenemos". Tales preguntas no pueden ser hechas ni en el grado, ni en la familia, ni en el chat, ni en el recreo. Es interesante ver los espacios que ellos espontáneamente comparan con la filosofía: el aula, la familia, el chat y el recreo. Algunos son percibidos como radicalmente diferentes, como es el caso del aula, un tiempo y un espacio sobre determinados, en el que todo está codificado en términos de transmisión de conocimientos. Nadie puso en duda que las preguntas y la conversación -que ellos insisten en mencionar como dos características fundamentales de la filosofía-, en el salón de clases adquieren otra naturaleza, que no le permite a los niños reconocerse en ellas. La familia, para Matheus Augusto, parece estar en la misma condición que el aula. No es así para Wesley, quien intuye que con su abuela podría conversar del mismo modo y de las mismas cosas que en nuestras experiencias. El problema para él es que faltaría gente. La filosofía se hace con preguntas, se hace conversando y se hace con otros, con muchos. Con una persona sola, para él es inconcebible. Tanto que le parece más importante la intervención de varias personas en el ejercicio de formular preguntas y conversar sobre ellas, que la presencia física de las mismas. Manteniendo esas condiciones, hasta el MSN podría adquirir un carácter filosófico. Matheus Augusto llama la atención sobre una diferencia que se da entre nuestras conversaciones y las del MSN. Aquellas parecen tratar sobre asuntos comunes: las chicas y el fútbol, por ejemplo. Las nuestras parecen ser el espacio más adecuado para formular en voz alta las preguntas que se hace a sí mismo. El recreo parece tener algo del tiempo de la filosofía. Allí se conversa sin interferencias de tareas y demandas escolares, pero se conversa de otra forma, que no llega a tornar filosófico lo que se dice, según plantean Arthur y Esther.

Entonces, ¿qué es que lo que se produce en los encuentros de filosofía que los torna un espacio y un tiempo diferentes de los otros por los cuales transitan estos niños? Aparentemente, en él las preguntas tienen un lugar central, pero, según sugieren, no cualquier pregunta. Las preguntas que hacen que la conversación se torne filosófica son aquellas que "salen de nuestra mente" para "pensar con ellas", para "aprender a discutir". La discusión será filosófica si "las preguntas aumentan", si podemos "juzgar las preguntas que tenemos en la cabeza", si se permite "que crezcan las dudas y las respuestas", si podemos "pensar más fácil" con la ayuda de los otros, si a partir de ellas "aprendemos cosas que ni imaginábamos".

En la introducción de la Historia de la sexualidad 2. El uso de los placeres, Michel Foucault (1986) define la filosofía como un "ejercicio de sí en el pensamiento". La filosofía para él nada tiene a ver con la legitimación de lo que ya se sabe, sino con aventurarse en el ejercicio de explorar los límites, con la posibilidad de pensar distinto de lo que se piensa, con abandonar el territorio seguro de lo ya sabido. En el mismo texto cuenta sobre el motivo que lo llevó a escribir el libro y lo atribuye a la curiosidad.

Es la curiosidad, esa única especie de curiosidad por lo demás, que vale la pena practicar con cierta obstinación: no la que busca asimilar lo que conviene conocer, sino la que permite alejarse de sí mismo. [Y continúa] De qué valdría el encarnizamiento del saber si solo hubiera de asegurar la adquisición de conocimientos y no, en cierto modo, y hasta donde se puede, el extravío del que conoce? (pp. 11-12).

Esa curiosidad, practicada con obstinación por Foucault es la curiosidad que permite encontrarse con cosas que ni se imaginaban. A la curiosidad no 
le es suficiente la información. Ella permanece ajena a la lógica de lo que conviene conocer, de lo que se puede prever según un tiempo programado. Las cosas que "ni imaginábamos" no pueden ser planeadas, no pueden ser anticipadas. Solo se puede anticipar lo que ya se conoce. Esa curiosidad produce un movimiento contrario, nos aleja de lo que somos y de lo que ya sabemos. No es un alejamiento que significa dar la espalda al mundo que nos rodea, sino uno que permite explorar otras posibilidades que habitan en él y otras maneras de habitarlo. Es una curiosidad que se inscribe en un tiempo diferente de aquel que se lanza como una flecha hacia el futuro en busca de lo previsible y de lo conveniente.

Hablando desde un campo diferente, el de la literatura, específicamente el de la literatura fantástica -un campo especializado en lidiar con cosas que "ni se imaginaban"-, Todorov (1975) afirma que los tópicos propios de lo fantástico no tienen que a ver con un tipo de acciones particulares, sino con una posición, con una percepción antes que con una interacción. La vacilación, la ambigüedad, la dificultad para atribuir fácilmente una sólida explicación a los acontecimientos extraordinarios resultan fundamentales para la noción de lo fantástico. Este se inscribe en un tiempo de incertezas. Quiere decir que los temas que dan cuerpo a este género más que por un contenido específico se caracterizan, básicamente, por un tipo de relación entre el hombre y el mundo.

En la literatura fantástica, el tiempo y el espacio no son el tiempo y el espacio de la vida cotidiana. En ella el tiempo y el espacio habituales son alterados, pero esa alteración se produce básicamente por una transformación de la mirada. El acceso a lo extraordinario se produce por un cambio de visión. "La visión pura y simple nos descubre un mundo llano, sin misterios. La visión indirecta es la única vía para lo maravilloso" (Todorov, 1975, p. 130). El autor explora la familiaridad entre las palabras maravilla (en francés merville), mirar (mirer) y espejo (miroir) y establece una relación con la frecuente presencia de aparatos ópticos en la literatura fantástica. Ellos no tendrían la función de tornar más transparente la mirada, de vincular de una forma más precisa el ojo y un punto particular del espacio. Antes, ellos vienen a materializar una especie de transgresión de la mirada. El visionario será "aquel que ve y no ve, al mismo tiempo grado superior y negación de la visión" (p. 131). Lo fantástico se presenta aquí como una forma de establecer un vínculo con el mundo que se funda sobre el extrañamiento de una mirada que deja de ver lo mismo de siempre cuando el tiempo y el espacio habituales son alterados y cuestionados.
Dejemos el pensamiento de Todorov resonando y volvamos al campo de la filosofía para continuar buscando ideas que nos ayuden a explorar eso que los niños de quinto grado consideran como lo propio del filosofar en la escuela. Bergson (2006b) afirma que el trabajo de la filosofía no está en la construcción de sistema, sino en la decisión de mirar para sí y en torno de sí con cierta ingenuidad. Critica a aquellos que creyeron, como Platón, que el descubrimiento de lo verdadero implica un movimiento del espíritu que se desprende, que escapa de las apariencias de acá abajo para apegarse a las realidades allá arriba; aquellos que pensaron que la tarea del metafísico consiste en dar la espalda a la vida práctica y transportarse a otro mundo. Al contrario, afirma que el trabajo del metafísico está en "continuar mirando lo que todo el mundo mira" (p. 160). Pero, ese mirar no implica ver lo que todo el mundo ve, al estar absorbido por los intereses prácticos cotidianos. Esa cierta ingenuidad de la mirada consiste, precisamente, en educar la atención en el sentido de "retirarle sus anteojos, de deshabituarla a las restricciones que las exigencias de la vida práctica le imponen" (p. 160). Posteriormente, Merleau-Ponty (1994, p. 19) reafirma: "La verdadera filosofía es reaprender a ver el mundo".

Esa curiosidad de Foucault, ese reaprendizaje de Merleau-Ponty, esa posibilidad de explorar "preguntas que tenemos y no sabemos" según Yasmín, esa experiencia de sentir que "las preguntas aumentan" en la perspectiva de Paulo, parece tener que ver con una particular forma de relacionarse con el tiempo. ¿Cómo pensar un tiempo en el cual es posible continuar mirando el mismo mundo que todo el mundo mira y, sin embargo, distinguir los desvíos que en él habitan? ¿Qué tiempo es ese en el cual pueden aflorar preguntas que nos habitan, pero que desconocemos? ¿dónde se pueden aprender cosas que ni se imaginaban?

Nuevamente Bergson nos auxilia, ahora con la insistencia en mostrar que el tiempo es diferente de ese que los calendarios y los relojes pretenden controlar, dividiendo momentos exactamente iguales y ordenándolos meticulosamente en el espacio en una sucesión de puntos abstractos y vacíos. Ese es un tiempo mensurable estructurado por la inteligencia en función de intereses prácticos. Pero hay un tiempo puro, como él lo llama, un tiempo cualitativo que la representación no consigue capturar. Es un tiempo que no se deja separar, dividir, fijar (Bergson, 1988).

Nuestra vida transcurre sin distinciones, afirma el filósofo (2006a). Estas son establecidas por nuestras interpretaciones. Sujetos a nuestra necesidad de actuar delimitamos nuestra percepción, restringimos

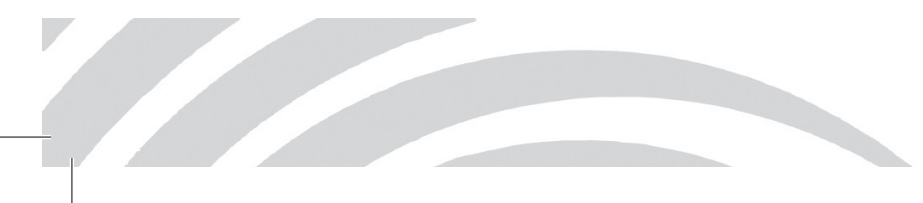


nuestro campo seleccionando, entre las múltiples posibilidades que existen en este mundo, apenas aquellas que se ajustan a nuestros intereses actuales. De un mundo múltiple solo conseguimos percibir aquello que nos es de utilidad. Así, descartamos las infinitas dimensiones de nuestra experiencia que conviven con nosotros todo el tiempo, que nos acompañan en cada paso que damos mientras nosotros consideramos que la única realidad posible es aquella que recortamos en función de las necesidades que demanda nuestro accionar. En esa mecánica solo hay lugar para la legitimación de las cosas que ya sabíamos, que ya imaginábamos.

Pero esa realidad virtual que descartamos es la que nos ofrece la posibilidad de enriquecer nuestro estar en el mundo. Al aflojar la red por la cual filtramos solo aquello que está ligado a la acción, podemos hacernos permeables a esas imágenes que fueron desconsideradas e invitarlas a componerse con nuestro presente. Entonces, nuestra realidad actual podrá ser más o menos rica dependiendo del grado de tensión o distensión de nuestros intereses.

Cuando la conciencia más tensa está en relación a la acción, más restringida se encuentra nuestra capacidad de percepción de la variedad que el mundo ofrece. Y, cuando está más distendida, más apartada de los intereses prácticos, se aproxima al sueño, dice Bergson (2006a). Cuando soñamos esa dimensión virtual que nos acompaña emerge de un modo diferente. Cuando el impulso que nos empuja al futuro se debilita, el paisaje que percibimos muda substancialmente.

Tal vez, podamos pensar que esa mudanza de paisaje que se produce cuando el tiempo no está sometido a los intereses prácticos (que hacen que el presente solo tenga sentido en función de un tiempo futuro) es la que los niños perciben en los encuentros de filosofía. ¿Será por eso que Sebastião siente que en ellos "nos relajamos", y que cuando salimos de allí "no nos relajamos más"? Nos atrevemos a considerar que lo que se relaja allí es el tiempo. Cuando conversamos; cuando nuestras preguntas aparecen no para encontrar respuestas sino para que nuestras dudas crezcan y se vuelvan más complejas, más sutiles; cuando podemos llegar a pensar "cosas que ni imaginábamos", el tiempo de nuestra conversación renuncia a programarse en función de intereses prácticos, desiste de objetivos que definan previamente su futuro y se aproxima al tiempo del sueño. Quizá por eso guarde una cierta semejanza con el tiempo del recreo, un tiempo que distiende la linealidad cronológica que sustenta la dinámica escolar.

Volvamos a la cuestión del cuidado en la práctica de la filosofía con niños, que planteábamos en el ini- cio. Consideramos que es principalmente del tiempo que se debe cuidar, si pretendemos embarcarnos en la experiencia colectiva de pensar. Más precisamente de ese tiempo que no se mide. Cuidar del tiempo, o mejor, de las condiciones para esperar que ese tiempo irrumpa. Cuidar de ese tiempo y de ese lugar donde nacen las ideas que no tienen compromiso con un resultado predeterminado, que no responden a intereses prácticos o a un futuro pre-visto, previsualizado. No cuidar del contenido de las ideas -ese puede cambiar-. Cuidar, precisamente, del lugary del tiempo donde nacen las ideas, el lugar y el tiempo que les permite transformarse. Cuidar de ese momento en que lo extraordinario puede ser común y lo común puede tornarse extraordinario (como en los sueños, como en el juego). Cuidar de la obstinación de esa curiosidad que nos permite extraviarnos, apartarnos de lo que ya sabemos.

Ese tiempo es de una naturaleza diferente a la del tiempo de la fabricación. No responde a causas y efectos. No es previsible. No es posible producirlo como se fabrica algo. Pero, es posible percibir sus efectos cuando irrumpe. Ese tiempo necesita que todos los que allí estamos (niños o adultos), estemos enteros para poder acoger silenciosamente nuestras preguntas, para que alguien pueda preguntarse en voz alta “¿por qué existen las estrellas?”, para tomar en serio la complejidad que esas cuestiones condensan y acompañarlas sin matarlas, sin sacarles la fuerza que las impulsa, para dejar que distintas voces se compongan dentro de ellas, que las transformen, las traduzcan, las superpongan a otras preguntas, y que las compongan con algunas afirmaciones. ¿Será que en eso consiste "llegar al punto de nuestras preguntas", como decía Ricardo?

Para que una experiencia de pensamiento con otros sea posible debemos cuidar de ese tiempo en el cual somos los mismos de siempre y, al mismo tiempo, podemos permitirnos pensar lo que no pensamos siempre. Debemos cuidar de la cualidad de ese tiempo, que es frágil y fácilmente digerible por la lógica de la mensura. Ese tiempo es delicado y por eso requiere una sutil sensibilidad para percibirlo, mucha paciencia para esperarlo y mucho cuidado para no matarlo. Si lo medimos con relojes, a veces, dura segundos, pero segundos que harán que haya valido la pena; a veces se resiste a presentarse y sentiremos que esa hora que pasamos hablando, dibujando o escribiendo habrá pasado en vano. Es ese tiempo -donde se aprenden cosas que no sirven para pasar de grado y que nadie enseña- el que nos muestra que nuestro mundo implacable, como nos decía Bioy Casares (1999), puede revelarnos que en él abundan cosas maravillosas. Quizá en esos 
segundos intempestivos se pueda encontrar una cierta explicación a la insistente resistencia de un grupo de alumnos de una escuela pública a perder su clase de filosofía.

\section{Referencias bibliográficas}

Bergson, H. (1988). Ensaio sobre os dados imediatos da consciência. Lisboa: Ediciones 70.

Bergson, H. (1999). Matéria y Memória. Ensaio sobre a relação do corpo com o espírito. São Paulo: Martins Fontes.

Bergson, H. (2006a). A lembrança do presente e o falso reconhecimento. (Traducción de Jonas Gonçalves Coelho). Trans/Form/Ação. 29(1). Disponible en: http://www. scielo.br/scielo.php?script=sci_arttext\&pid=S0101$31732006000100007 \& \operatorname{lng}=$ pt\&nrm=iso $>$.

Bergson, H. (2006b). O pensamento e o movente. São Paulo: Martins Fontes.

Bioy C., A. (1999). De las cosas maravillosas. Buenos Aires: Temas.

Deleuze, G. (1999). Bergsonismo. São Paulo: Editora 34.

Foucault, M. (1986). La historia de la sexualidad 2. El uso de los placeres. México: Siglo XXI.

Kennedy, D. (1997). Las cinco comunidades. En W. Kohan y V. Waskman (orgs.) ¿Qué es filosofía para niños?: ideas y propuestas para pensar la educación. Buenos Aires: Oficina de Publicaciones del CBC - Universidad de Buenos Aires.

Merleau-Ponty, M. (1994). Fenomenologia da percepção. São Paulo: Martins Fontes.

Todorov, T. (1975). Introdução à literatura fantástica. São Paulo: Perspectiva. 